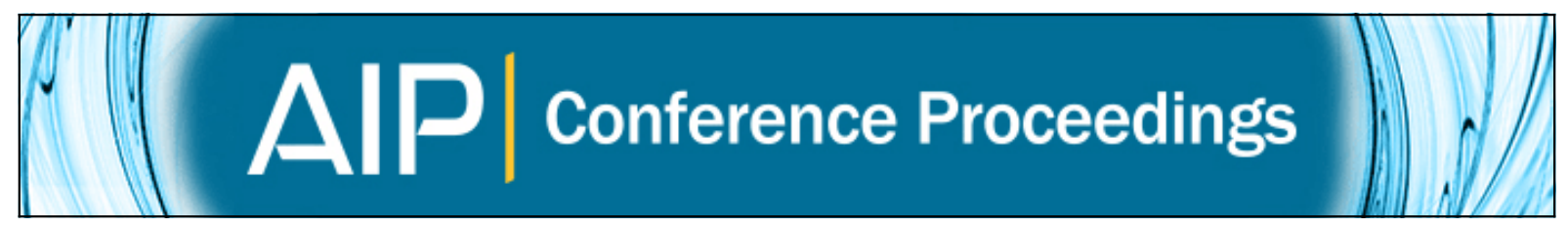

\title{
SuperCDMS status from Soudan and plans for SNOLab
}

J. Sander, Z. Ahmed, A. J. Anderson, S. Arrenberg, D. Balakishiyeva, R. B. Thakur, D. A. Bauer, D. Brandt, P. L. Brink, R. Bunker, B. Cabrera, D. O. Caldwell, D. G. Cerdeno, H. Chagani, J. Cooley, B. Cornell, C. H. Crewdson, P. Cushman, M. Daal, P. C. F. Di Stefano, E. Do Couto E Silva, T. Doughty, L. Esteban, S. Fallows, E. FigueroaFeliciano, J. Fox, M. Fritts, G. L. Godfrey, S. R. Golwala, J. Hall, J. Hasi, S. A. Hertel, T. Hofer, D. Holmgren, L. Hsu, M. E. Huber, A. Jastram, O. Kamaev, B. Kara, M. H. Kelsey, P. Kim, M. Kiveni, K. Koch, M. Kos, S. W. Leman, S. Liu, B. Loer, R. Mahapatra, V. Mandic, C. Martinez, K. A. Mccarthy, N. Mirabolfathi, R. Moffat, D. C. Moore, P. Nadeau, R. Nelson, K. Page, R. Partridge, M. Pepin, A. Phipps, K. Prasad, M. Pyle, H. Qiu, X. Qiu, R. Radpour, W. Rau, A. Reisetter, R. W. Resch, Y. Ricci, T. Saab, B. Sadoulet, R. W. Schnee, S. Scorza, B. Serfass, B. Shank, K. Shneck, D. Speller, K. M. Sundqvist, A. N. Villano, B. Welliver, S. Yellin, J. Yen, J. Yoo, B. A. Young, and J. Zhang

Citation: AIP Conference Proceedings 1534, 129 (2013); doi: 10.1063/1.4807350

View online: http://dx.doi.org/10.1063/1.4807350

View Table of Contents: http://scitation.aip.org/content/aip/proceeding/aipcp/1534?ver=pdfcov Published by the AIP Publishing

\section{Articles you may be interested in}

Background considerations for SuperCDMS

AIP Conf. Proc. 1549, 223 (2013); 10.1063/1.4818113

Status and future of the CDMS experiment: CDMS-II to SuperCDMS AIP Conf. Proc. 957, 193 (2007); 10.1063/1.2823758

Remarks on search methods for stable, massive, elementary particles AIP Conf. Proc. 596, 156 (2001); 10.1063/1.1426799

Gamma rays from dark matter AIP Conf. Proc. 558, 491 (2001); 10.1063/1.1370815

Status of the CDMS search for WIMPs AIP Conf. Proc. 478, 378 (1999); 10.1063/1.59416 


\section{SuperCDMS Status from Soudan and Plans for SNOLab}

J. Sander*, Z. Ahmed ${ }^{\dagger}$, A.J. Anderson**, S. Arrenberg ${ }^{\ddagger}$, D. Balakishiyeva ${ }^{\S}$, R.B. Thakur "I, D.A. Bauer ${ }^{\mathbb{I l}}$, D. Brandt" ${ }^{\|}$, P.L. Brink ${ }^{\|}$, R. Bunker ${ }^{\dagger \dagger}$, B. Cabrera ${ }^{\ddagger}$, D.O. Caldwell ${ }^{\S \S}$, D. G. Cerdeno ${ }^{\text {qाII }}$, H. Chagani***, J. Cooley ${ }^{\dagger \dagger \dagger}$, B. Cornell ${ }^{\dagger}$, C.H. Crewdson ${ }^{* \neq}$, P. Cushman***, M. Daal ${ }^{\S \S \S}$, P.C.F. Di Stefano ${ }^{* *}$, E. Do Couto E Silvall, T. Doughty ${ }^{\S \S}$, L. Esteban ${ }^{\text {IIIII }}$, S. Fallows ${ }^{* * *}$, E. Figueroa-Feliciano**, J. Fox ${ }^{\ddagger \neq}$, M. Fritts***, G.L. Godfrey ${ }^{\|}$, S.R. Golwala ${ }^{\dagger}$,

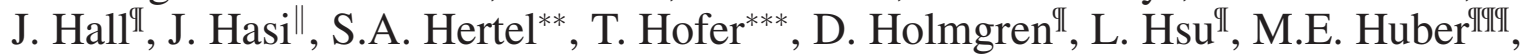

A. Jastram*, O. Kamaev ${ }^{\ddagger \neq}$, B. Kara ${ }^{\dagger \dagger \dagger}$, M.H. Kelsey\|, P. Kim", M. Kiveni ${ }^{\dagger \dagger}$, K. Koch*, M. Kos ${ }^{\dagger \dagger}$, S.W. Leman**, S. Liu ${ }^{* *}$, B. Loer ${ }^{\mathbb{I l}}$, R. Mahapatra*, V. Mandic***, C. Martinez ${ }^{\ddagger *}$, K.A. Mccarthy ${ }^{* *}$, N. Mirabolfathi ${ }^{\S \S \S}$, R. Moffat ${ }^{\dagger *}$, D.C. Moore ${ }^{\dagger}$, P. Nadeau ${ }^{\ddagger \neq}$, R. Nelson ${ }^{\dagger}$, K. Page ${ }^{\dagger+}$, R. Partridge ${ }^{\|}$, M. Pepin***, A. Phipps ${ }^{\S \S \S}$, K. Prasad ${ }^{*}$, M. Pyle ${ }^{\S \S}$, H. Qiu ${ }^{\dagger \dagger \dagger}$, X. Qiu***, R. Radpour ${ }^{* * *}$, W. Rau ${ }^{\ddagger *}$, A. Reisetter, R.W. Resch" ${ }^{\|}$Y. Ricci ${ }^{\ddagger \sharp}$, T. Saab ${ }^{\S}$, B. Sadoulet ${ }^{\S \S}$, R.W. Schnee ${ }^{\dagger \dagger}$, S. Scorza ${ }^{\dagger \dagger}$, B. Serfass ${ }^{\S \S \S}$, B. Shank ${ }^{\ddagger \neq}$, K. Shneck ${ }^{\|}$, D. Speller ${ }^{\S \S}$,

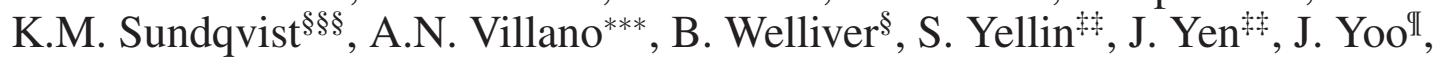
B.A. Young and J. Zhang ${ }^{* * *}$

*Department of Physics, Texas A\&M University, College Station, TX 77843, USA

${ }^{\dagger}$ Division of Physics, Mathematics, and Astronomy, California Institute of Technology, Pasadena, CA 91125, USA

${ }^{* *}$ Department of Physics, Massachusetts Institute of Technology, Cambridge, MA 02139, USA

${ }^{\ddagger}$ Physics Institute, University of Zrich, Winterthurerstr. 190, CH-8057, Switzerland

${ }^{\S}$ Department of Physics, University of Florida, Gainesville, FL 32611, USA

"Fermi National Accelerator Laboratory, Batavia, IL 60510, USA

"ISLAC National Accelerator Laboratory/Kavli Institute for Particle Astrophysics and Cosmology, 2575 Sand Hill Road, Menlo Park 94025, CA

${ }^{\dagger}$ Department of Physics, Syracuse University, Syracuse, NY 13244, USA

\$Department of Physics, Stanford University, Stanford, CA 94305, USA

${ }^{\S}$ Department of Physics, University of California, Santa Barbara, CA 93106, USA

IIII Departamento de

FísicaTeóricaandInstitutodeF ísicaTeóricaUAM/CSIC, UniversidadAutónomadeMadrid, 28049Madrid, Spain

*** School of Physics \& Astronomy, University of Minnesota, Minneapolis, MN 55455, USA

${ }^{\dagger \dagger}$ Department of Physics, Southern Methodist University, Dallas, TX 75275, USA

\$D Department of Physics, Queen's University, Kingston ON, Canada K7L 3N6

$\S \S$ Department of Physics, University of California, Berkeley, CA 94720, USA

IITI Department of Physics, University of Colorado, Denver, CO 80217, USA

Department of Physics, University of Evansville, Evansville, IN 47722, USA

Department of Physics, Santa Clara University, Santa Clara, CA 95053, USA

Workshop on Dark Matter, Unification and Neutrino Physics

AIP Conf. Proc. 1534, 129-135 (2013); doi: 10.1063/1.4807350

(C) 2013 AIP Publishing LLC 978-0-7354-1158-6/\$30.00 


\begin{abstract}
Matter, as we know it, makes up less than 5\% of the Universe. Various astrophysical observations have confirmed that one quarter of the Universe and most of the matter content in the Universe is made up of Dark Matter. The nature of Dark Matter is yet to be discovered and is one of the biggest questions in Physics. Particle Physics combined with astrophysical measurements of the abundance gives rise to a Dark Matter candidate called Weakly Interacting Massive Particle (WIMP). The low density of WIMPs in the galaxies and the extremely weak nature of the interaction with ordinary matter make detection of the WIMP an extraordinarily challenging task, with abundant fakes from various radioactive and cosmogenic backgrounds with much stronger electromagnetic interaction. The extremely weak nature of the WIMP interaction dictates detectors that have extremely low naturally occurring radioactive background, a large active volume (mass) of sensitive detector material to maximize statistics, a highly efficient detector based rejection mechanism for the dominant electromagnetic background and sophisticated analysis techniques to reject any residual background. This paper describes the status of the SuperCDMS experiment.
\end{abstract}

Keywords: CDMS, dark matter, WIMP, direct detection

PACS: $14.80 . \mathrm{Ly}, 95.35 .+\mathrm{d}, 95.30 . \mathrm{Cq}, 95.30 .-\mathrm{k}, 85.25 . \mathrm{Oj}, 29.40 . \mathrm{Wk}$

\title{
INTRODUCTION
}

Never before have we had such a convergence of various astronomical measurements to provide us with a "standard model" of the Universe. The last decade has brought about stunning measurements of the fluctuations in cosmic microwave background, expansion of the Universe as seen through supernovae, large and small scale structure through lensing. The "standard model" places us in the most awkward situation of being certain about the nature of less than $5 \%$ of the content of the Universe.

A variety of cosmological observations $[1,2]$ indicate that $80 \%$ of the matter in the Universe is nonbaryonic and dark, presumably in the form of elementary particles produced in the early Universe. Because no such particles have yet been identified in particle accelerators, these observations require new fundamental particle physics. Weakly Interacting Massive Particles (WIMPs) are a particularly interesting generic class of candidates for this dark matter [3, 4] because independent arguments from cosmology and particle physics converge on the same conclusion. A WIMP is generically defined as a massive particle created in the early universe that couples via a weak-scale interaction, allowing it to decouple and stop annihilating when non-relativistic. A weak-scale annihilation cross section naturally results in the relic density required of nonbaryonic dark matter. Simultaneously, new particle physics at the $W$ and $Z$ scale is required to solve the "hierarchy problem" by canceling radiative corrections that would push the Higgs mass higher than precision electroweak data indicate. The most popular solution, supersymmetry, naturally yields a WIMP in the form of the lightest superpartner (LSP). Thus, searches for astrophysical dark matter particles seek to solve fundamental problems in both cosmology and particle physics and complement accelerator searches for physics beyond the Standard Model.

\section{DIRECT DETECTION}

The goal of direct detection experiments is to detect the signature of WIMPs recoiling on their terrestrial detectors. The expected rate of interaction of the WIMP on a terrestrial detector is less than 1 per $10 \mathrm{~kg}$-days [5]. The expected background rate from radioactivity in surrounding material is expected to be more than a million times larger. Hence, background rejection is critical and the goal is to employ shielded detectors with strong background rejection capabilities. Shielding must block the radioactive background as well as cosmic-induced backgrounds, which can produce neutrons which are difficult to distinguish from WIMPs.

To reject electromagnetic backgrounds, detectors are designed to take advantage of the different natures of the recoil of electromagnetic background and the neutral WIMPs by intercomparing two signal types. SuperCDMS observes phonons sensors and ionization electrodes. The nature of the recoil is usually significantly different in the ionization signal, since nuclear recoil from the signal WIMP or neutron deposits the recoil energy in a very small distance due to their collision with the nucleus, leading to very dense ionization density and low ionization efficiency. Electron recoils from radioactive background leads to sparse ionization density, in which the collision is with orbital electron with efficient ionization. Measuring the ionization energy or its equivalent can allow electromagnetic interactions to be differentiated from nuclear recoils.

In addition, the WIMP recoil not only depends on how much total mass of the detector, but may also depend on the exact nature of the nuclear composition of the detector target. Because they are expected to be galactically 

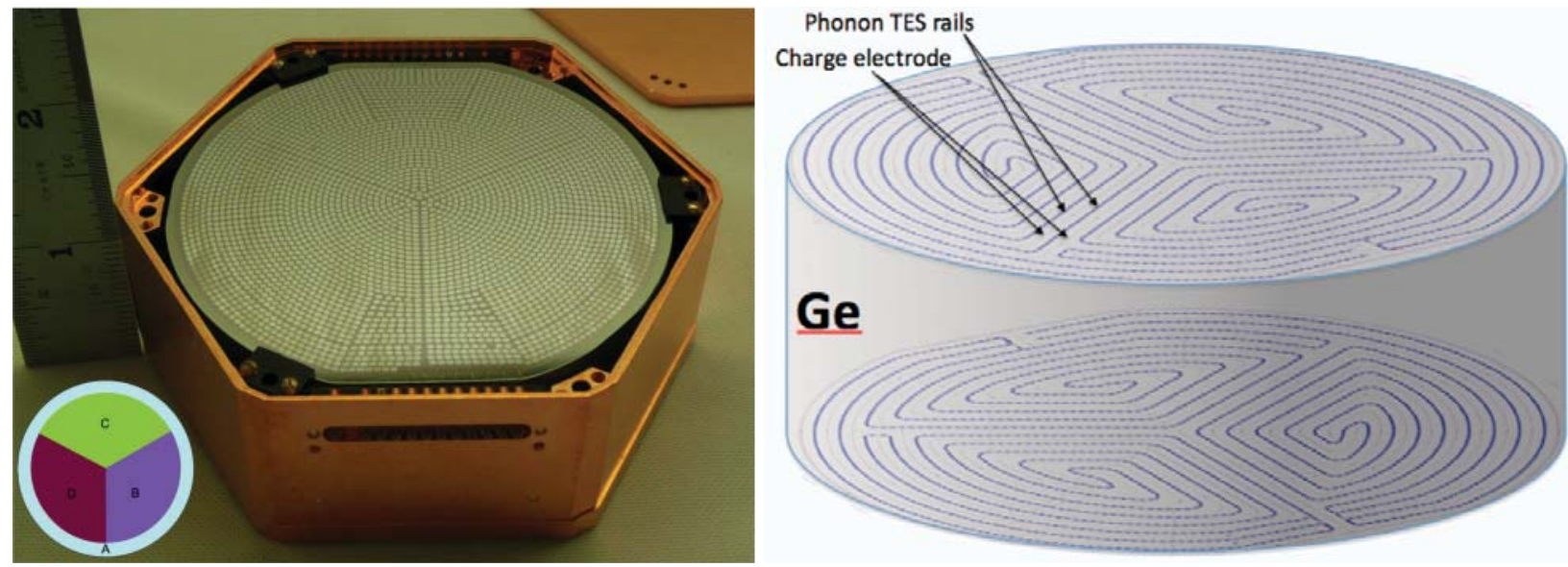

FIGURE 1. Left: A SuperCDMS iZIP detector. Right: A schematic view of the iZIP phonon and charge sensor layout.

bound, WIMPs are slow moving and are expected to undergo coherent elastic scattering on the entire nucleus, which tremendously enhances the interaction rate, providing a boost to the rate that is proportional to the fourth power of the atomic number [5].

\section{SUPERCDMS}

SuperCDMS employs detectors composed of high purity Ge crystals with a diameter of 76mm and a thickness of $25 \mathrm{~mm}$ and is currently taking WIMP-search data in the Soudan Underground Laboratory using 15 Ge detectors for a total target mass of about $10 \mathrm{~kg}$. Four phonon and two ionization sensors are photolithographically fabricated in each of the two detector faces. When a particle interaction takes place in the detector, electron-hole pairs and phonons are created. The phonons propagate through the crystal and are collected in the phonon sensors. Electrons and holes created by an event are drifted through the crystal following an applied field of about $1.5 \mathrm{~V} / \mathrm{cm}$ and collected on the detector faces. The interleaving of the ionization and phonon sensors is reflected in the detectors' name, Interleaved Z-dependent Ionization and Phonon detectors (iZIPs). To define a clear fiducial volume, a phonon sensor and an ionization sensor span the outer detector region while the remaining sensors span the inner detector region.

The ratio of the observed ionization signal to phonon signal is termed yield and is the primary handle for discriminating between bulk electromagnetic recoils and nuclear recoils. The low ionization efficiency of nuclear recoils results in a yield that is about three times lower than the typical yield of a bulk electromagnetic event with unity yield. The separation between bulk electromagnetic events and nuclear recoils has been shown to be at least at the level of $5 \sigma$ for recoil energies above $10 \mathrm{keV}_{n r}$.

The iZIP detector is designed to provide potent rejection for electromagnetic interactions occurring on detector surfaces. These surface events were the dominant background faced by CDMS due to their reduced ionization. Figure 1 shows an iZIP detector (left) and a schematic view of the interdigitated electrode structure used to reject surface events. Events occurring on a detector face undergo carrier transport along the face resulting in highly asymmetric ionization signals observed by the electrodes on the two detector faces.

While the efficacy of iZIP surface event rejection was demonstrated at the UC Berkeley test facility, iZIP performance is demonstrated in situ at Soudan. In order to obtain the number of surface events required to demonstrated the impressive iZIP surface event rejection, two detectors were installed with a ${ }^{210} \mathrm{~Pb}$ source. The ${ }^{210} \mathrm{~Pb}$ decay chain is the primary source of SuperCDMS surface events. The rate of surface events produced by each source is approx70 events/hr. Figure 2 shows that in addition to having low yield, iZIP surface events have ionization signals that are asymmetric between electrodes on the detector faces.

During about 900 hours of live time, about $8 \times 10^{4}$ surface events were obtained and shown in Figure 2. No events were observed within the fiducial volume. The $90 \%$ confidence upper limit on the surface event background rate is $2.9 \times 10^{-5}$. Additionally the fraction of the crystal encompassed by the fiducial volume is $63 \%$ or nearly twice that of 

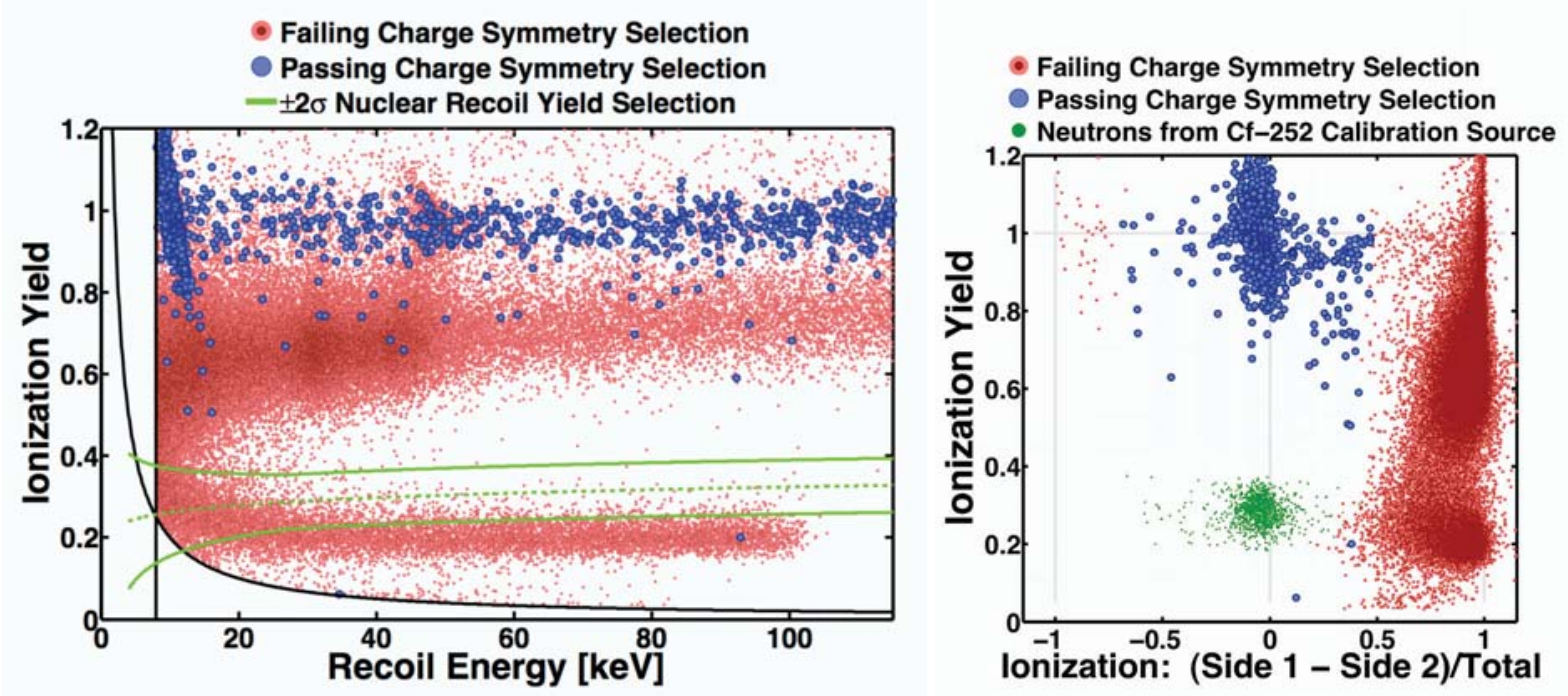

FIGURE 2. Left: Surface events (red) have reduced ionization compared to bulk electromagnetic events (blue). The $2 \sigma$ nuclear recoil band, or WIMP signal region, is defined from neutron-induced nuclear recoils due to illuminating the detectors with a ${ }^{252} \mathrm{Cf}$ source. Most surface events fall outside the signal region. The band of surface events with a yield of about 0.2 are due to recoiling ${ }^{206}$ Po nuclei from ${ }^{210}$ Po alpha decay. The vertical line at $8 \mathrm{keV}$ indicates the analysis threshold. Right: Surface events on side 1 of the detector have asymmetric observed ionization energy unlike bulk electromagnetic events or nuclear recoils (green).

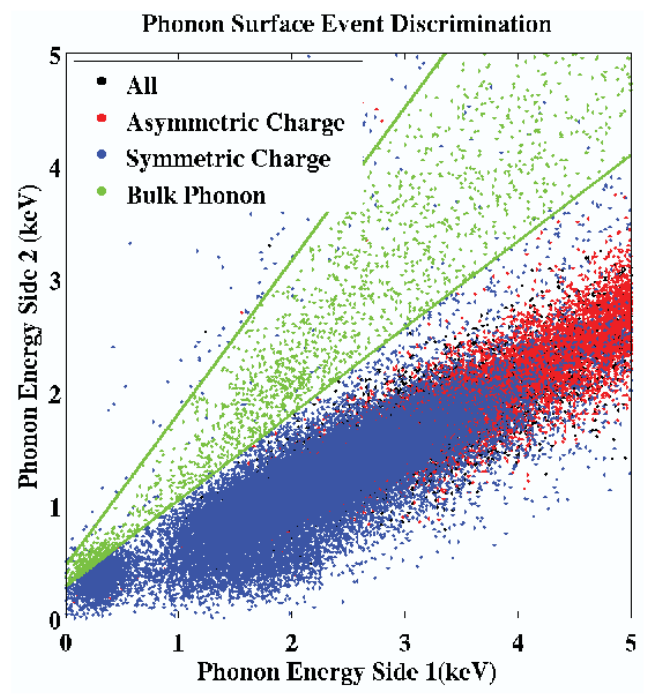

FIGURE 3. Phonon energy observed by the sensors on the two faces of an iZIP. Bulk nuclear recoils (green) can be distinguished from surface events down to $2 \mathrm{keV}$.

the CDMSII detectors. An iZIP then has about 4-5 greater sensitive volume than a CDMSII detector after accounting for the iZIP's greater mass.

The partition of energy between the phonon sensors on the detector faces also provides a potent handle for rejection surface events. Phonon sensor partition was not used to obtain the in situ surface event rejection numbers. Data from the UC Berkeley test facility shows how phonon partition can be used to reject surface events down to recoil energies 

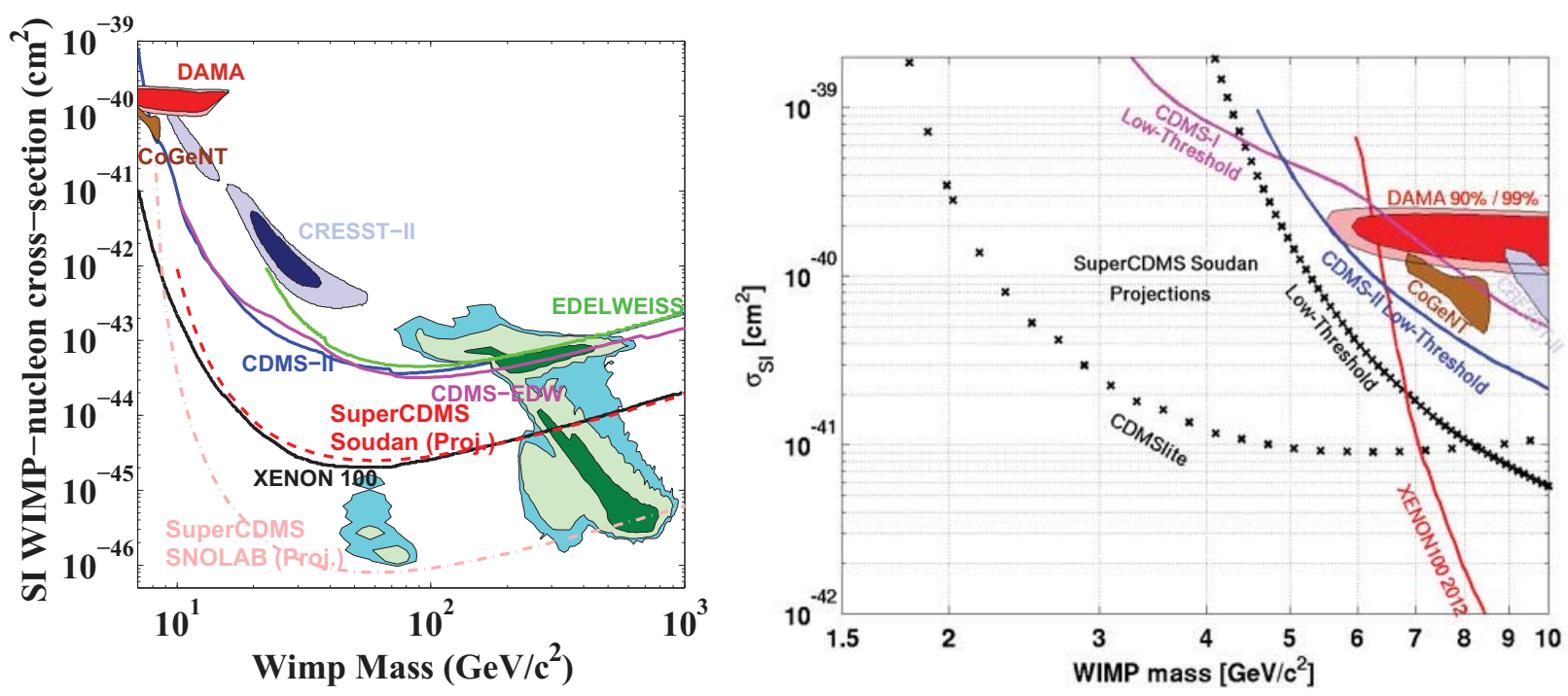

FIGURE 4. Projected SuperCDMS limits from Soudan and SNOLAB (dashed red) with 4 years operationg a 200kg Ge payload. Left: Projected limits are compared with CDMSII Soudan [6, 7] (blue solid), EDELWEISS II [8] (green solid), combined CDMSEDELWEISS II [9](magenta), and XENON100 [10] (black solid). The blue filled region indicates the region where CRESST II reports a signal [11]: 1-sigma allowed region (dark blue), 2 sigma allowed region (light blue). The red dotted portions of the graph indicate the regions where DAMA reports a signal [12]: 90\% C.L. (red), and 99\% C.L. (dark red). The colored regions show the current cMSSM regions (with recent LHC and Higgs constraints) predicting where WIMPs may be found, assuming flat priors: Strege et. al. [13], at 68\% (green), 95\% (light green), and 99\% (cyan) C.L. Right: Projected low mass limits (black).

as low as $2 \mathrm{keV}_{n r}$ as shown by Figure 3 .

R\&D for the planned move of SuperCDMS to SNOLAB is underway with a goal of beginning data taking in late 2016. The move creates the opportunity for numerous substantial improvements. This proceeding focuses on detector fabrication. In order to deliver order-of-magnitude increases in sensitivity, it is critical to deliver substantially increased target mass while achieving reduced background. The iZIP technology provides the reduced background; larger detectors and improved detector fabrication are key to delivering substantially increased target Ge mass. Cost scales with number of detectors, while WIMP sensitivity scales with scales with target mass in a low background environment. SuperCDMS Soudan proposes to employ 150 larger, $100 \mathrm{~mm}$ diameter by $33 \mathrm{~mm}$ thick Ge crystals for a total mass of $200 \mathrm{~kg}$. Improvements in detector fabrication, including the commissioning of Texas A\&M as a dedicated, highly-automated fabrication facility designed for the high-throughput requirements of a ton-scale Ge experiment, in addition to the primary fabrication facility, the Stanford Nanofabrication Facility, have led to the proposed target mass and provides numerous opportunities to cross-check detector performance. Both facilities have demonstrated the required throughput. Improvements in fabrication reliability show the potential to increase detector yield and reduce the number of hours required to test each detector.

Detectors will be employed in vertical stacks of 6. Improvements in noise performance, including using highelectron-mobility transistors (HEMPTs) are expected to allow the low mass analyses to be sensitive to recoils depositing energy as low as $1 \mathrm{keV}$. Figure 4 shows the resulting projected limits from SuperCDMS at Soudan and SuperCDMS SNOLAB.

\section{CONCLUSION}

CDMS has long been a leader in WIMP direct detection. The modular nature of the CDMS detectors combined with the iZIP testing at both the UC Berkeley test facility as well as at Soudan combine to provide the background control required for robust sensitivity projections. The proposed SuperCDMS SNOLAB experiment will have a reach comparable with other G2 experiments. Additionally, any potential WIMP signal will require a second experiment to 
confirm it. Likewise, multiple target nuclei will be required to begin to unravel the implications of a WIMP discovery. CMDS's history of delivered low-background results gives it unique ability to test and understand a potential signal.

\section{ACKNOWLEDGMENTS}

This work is supported in part by the National Science Foundation (Grant Nos. AST-9978911, PHY-0542066, PHY-0503729, PHY-0503629, PHY-0503641, PHY-0504224, PHY-0705052, PHY-0801708, PHY-0801712, PHY0802575, PHY-0847342, and PHY-0855525), by the Department of Energy (Contracts DE-AC03-76SF00098, DEFG02-91ER40688, DE-FG02-92ER40701, DE-FG03-90ER40569, and DE-FG03-91ER40618), by the Swiss National Foundation (SNF Grant No. 20-118119), and by NSERC Canada (Grant SAPIN 341314-07).

\section{REFERENCES}

1. E. Komatsu et al. for the WMAP collaboration, "Five-Year Wilkinson Microwave Anisotropy Probe (WMAP) Observations: Cosmological Interpretation,", astro-ph/0803.0547, submitted to Astroph. J. Suppl., 2008.

2. D. Tytler, J. M. O'Meara, N. Suzuki, and D. Lubin, "Review of Big Bang Nucleosynthesis and Primordial Abundances," Phys. Scr. T85, 12-31 (2000).

3. B. W. Lee and S. W. Weinberg, "Cosmological Lower Bound on Heavy Neutrino Masses," Phys. Rev. Lett. 39, 165-168 (1977).

4. G. Jungman, M. Kamionkowski, and K. Griest, "Supersymmetric Dark Matter," Phys. Rep. 267, 195-373 (1996).

5. J. D. Lewin and P F. Smith, "Review of mathematics, numerical factors, and corrections for dark matter experiments based on elastic nuclear recoil," Astropart. Phys. 6, 87-112 (1996).

6. Z. Ahmed, D. S. Akerib, S. Arrenberg, C. N. Bailey, D. Balakishiyeva, L. Baudis, D. A. Bauer, P. L. Brink, T. Bruch, R. Bunker, B. Cabrera, D. O. Caldwell, J. Cooley, E. do Couto e Silva, P. Cushman, M. Daal, F. DeJongh, P. Di Stefano, M. R. Dragowsky, L. Duong, S. Fallows, E. Figueroa-Feliciano, J. Filippini, J. Fox, M. Fritts, S. R. Golwala, J. Hall, R. Hennings-Yeomans, S. A. Hertel, D. Holmgren, L. Hsu, M. E. Huber, O. Kamaev, M. Kiveni, M. Kos, S. W. Leman, S. Liu, R. Mahapatra, V. Mandic, K. A. McCarthy, N. Mirabolfathi, D. Moore, H. Nelson, R. W. Ogburn, A. Phipps, M. Pyle, X. Qiu, E. Ramberg, W. Rau, A. Reisetter, R. Resch, T. Saab, B. Sadoulet, J. Sander, R. W. Schnee, D. N. Seitz, B. Serfass, K. M. Sundqvist, M. Tarka, P. Wikus, S. Yellin, J. Yoo, B. A. Young, and J. Zhang, "Results from a Low-Energy Analysis of the CDMS II Germanium Data" Phys.Rev.Lett. 106, 131302 (2011).

7. Z. Ahmed, D. S. Akerib, S. Arrenberg, C. N. Bailey, D. Balakishiyeva, L. Baudis, D. A. Bauer, P. L. Brink, T. Bruch, R. Bunker, B. Cabrera, D. O. Caldwell, J. Cooley, P. Cushman, M. Daal, F. DeJongh, M. R. Dragowsky, L. Duong, S. Fallows, E. Figueroa-Feliciano, J. Filippini, M. Fritts, S. R. Golwala, D. R. Grant, J. Hall, R. Hennings-Yeomans, S. A. Hertel, D. Holmgren, L. Hsu, M. E. Huber, O. Kamaev, M. Kiveni, M. Kos, S. W. Leman, R. Mahapatra, V. Mandic, K. A. McCarthy, N. Mirabolfathi, D. Moore, H. Nelson, R. W. Ogburn, A. Phipps, M. Pyle, X. Qiu, E. Ramberg, W. Rau, A. Reisetter, T. Saab, B. Sadoulet, J. Sander, R. W. Schnee, D. N. Seitz, B. Serfass, K. M. Sundqvist, M. Tarka, P. Wikus, S. Yellin, J. Yoo, B. A. Young, and J. Zhang, "Dark Matter Search Results from the CDMS II Experiment," Science Vol. 327, no. 5973:1619-1621, (2010), [arXiv:0912.3592].

8. E. Armengaud, C. Augier, A. Benoit, L. Berge, J. Blumer, A. Broniatowski, V. Brudanin, B. Censier, G. Chardin, M. Chapellier, F. Charlieux, P. Coulter, G.A. Cox, X. Defayd, M. De Jesus, Y. Dolgorouki, J. Domange, L. Dumoulin, K. Eitel, D. Filosofov, N. Fourches, J. Gascon, G. Gerbier, J. Gironnet, M. Grosa, S. Henry, S. Herve, A. Juillard, H. Kluck, V. Kozlov, H. Kraus, V.A Kudryavtsev, P. Loaiza, S. Marnieros, X.F. Navick, C. Nones, E. Olivieri, P. Pari, L. Pattavina, B. Paul, M. Robinson, S. Rozov, V. Sanglard, B. Schmidt, S. Scorza, S. Semikh, A.S. Torrento-Coello, L. Vagneron, M.A. Verdier, R.J. Walker, E. Yakushev, "Final results of the EDELWEISS-II WIMP search using a 4-kg array of cryogenic germanium detectors with interleaved electrodes," Phys. Lett. B 702:329-335, (2011), [arXiv:1103.4070].

9. Z. Ahmed, D. S. Akerib, E. Armengaud, S. Arrenberg, C. Augier, C. N. Bailey, D. Balakishiyeva, L. Baudis, D. A. Bauer, A. Benoit, L. Berge, J. Blumer, P. L. Brink, A. Broniatowski, T. Bruch, V. Brudanin, R. Bunker, B. Cabrera, D. O. Caldwell, B. Censier, M. Chapellier, G. Chardin, F. Charlieux, J. Cooley, P. Coulter, G. A. Cox, P. Cushman, M. Daal, X. Defay, M. De Jesus, F. DeJongh, P. C. F. Di Stefano, Y. Dolgorouki, J. Domange, L. Dumoulin, M. R. Dragowsky, K. Eitel, S. Fallows, E. Figueroa-Feliciano, J. Filippini, D. Filosofov, N. Fourches, J. Fox, M. Fritts, J. Gascon, G. Gerbier, J. Gironnet, S. R. Golwala, M. Gros, J. Hall, R. Hennings-Yeomans, S. Henry, S. A. Hertel, S. Herve, D. Holmgren, L. Hsu, M. E. Huber, A. Juillard, O. Kamaev, M. Kiveni, H. Kluck, M. Kos, V. Kozlov, H. Kraus, V. A. Kudryavtsev, S. W. Leman, S. Liu, P. Loaiza, R. Mahapatra, V. Mandic, S. Marnieros, C. Martinez, K. A. McCarthy, N. Mirabolfathi, D. Moore, P. Nadeau, X-F. Navick, H. Nelson, C. Nones, R. W. Ogburn, E. Olivieri, P. Pari, L. Pattavina, B. Paul, A. Phipps, M. Pyle, X. Qiu, W. Rau, A. Reisetter, Y. Ricci, M. Robinson, S. Rozov, T. Saab, B. Sadoulet, J. Sander, V. Sanglard, B. Schmidt, R. W. Schnee, S. Scorza, D. N. Seitz, S. Semikh, B. Serfass, K. M. Sundqvist, M. Tarka, A. S. Torrento-Coello, L. Vagneron, M.-A. Verdier, R. J. Walker, P. Wikus, E. Yakushev, S. Yellin, J. Yoo, B. A. Young, and J. Zhang, "Combined limits on WIMPs from the CDMS and EDELWEISS experiments," Phys. Rev. D84, 011102 (2011).

10. E. Aprile, K. Arisaka, F. Arneodo, A. Askin, L. Baudis, A. Behrens, K. Bokeloh, E. Brown, T. Bruch, G. Bruno, J. M. R. Cardoso, W.-T. Chen, B. Choi, D. Cline, E. Duchovni, S. Fattori, A. D. Ferella, F. Gao, K.-L. Giboni, E. Gross, A. Kish, C. W. 
Lam, J. Lamblin, R. F. Lang, C. Levy, K. E. Lim, Q. Lin, S. Lindemann, M. Lindner, J. A. M. Lopes, K. Lung, T. Marrodan Undagoitia, Y. Mei, A. J. Melgarejo Fernandez, K. Ni, U. Oberlack, S. E. A. Orrigo, E. Pantic, R. Persiani, G. Plante, A. C. C. Ribeiro, R. Santorelli, J. M. F. dos Santos, G. Sartorelli, M. Schumann, M. Selvi, P. Shagin, H. Simgen, A. Teymourian, D. Thers, O. Vitells, H. Wang, M. Weber, and C. Weinheimer, "Dark Matter Results from 100 Live Days of XENON100 Data," [arXiv: 1104.2549v2].

11. G. Angloher, M. Bauer, I. Bavykina, A. Bento, C. Bucci, C. Ciemniak, G. Deuter, F. von Feilitzsch, D. Hauff, P. Huff, C. Isaila, J. Jochum, M. Kiefer, M. Kimmerle, J.-C. Lanfranchi, F. Petricca, S. Pfister, W. Potzel, F. Probst, F. Reindl, S. Roth, K. Rottler, C. Sailer, K. Schaffner, J. Schmaler, S. Scholl, W. Seidel, M. von Sivers, L. Stodolsky, C. Strandhagen, R. Straus, A. Tanzke, I. Usherov, S. Wawoczny, M. Willers, and A. Zoller, "Results from $730 \mathrm{~kg}$ days of the CRESST-II Dark Matter Search," [arXiv:1109.0702].

12. D. Hooper, J.I Collar, J. Hall, D. McKinsey, and C.M. Kelso, "Consistent dark matter interpretation for CoGeNT and DAMA/LIBRA," Phys. Rev. D. 82, 123509 (2010).

13. C. Strege, G. Bertone, D. G. Cerdeno, M. Fornasa, R. Ruiz de Austri, R. Trotta, "Updated global fits of the cMSSM including the latest LHC SUSY and Higgs searches and XENON100 data," J. Cosmol. Astropart. Phys. 03, 030, (2012) [arXiv:1112.4192]. 